\title{
Young Scientists Need Emotional Support and a Framework When Drafting Scientific Articles
}

\author{
Jannie Laursen, ${ }^{1}$ Kristoffer Andresen, ${ }^{2}$ and Jacob Rosenberg ${ }^{2}$ \\ ${ }^{1}$ Department of Clinical Pharmacology, Bispebjerg Hospital, Copenhagen, Denmark \\ ${ }^{2}$ Department of Surgery, Herlev Hospital, University of Copenhagen, Herlev, Denmark \\ Correspondence should be addressed to Kristoffer Andresen; kristofferandresen@gmail.com
}

Received 4 September 2017; Accepted 27 November 2017; Published 14 December 2017

Academic Editor: P. Ravi Shankar

Copyright (c) 2017 Jannie Laursen et al. This is an open access article distributed under the Creative Commons Attribution License, which permits unrestricted use, distribution, and reproduction in any medium, provided the original work is properly cited.

\begin{abstract}
Introduction. When teaching young scientists to write scientific articles, it is important to consider several aspects of learning including intrinsic motivation, since the scientific work can be demanding in a different way than routine clinical work. The aim of this study was to investigate young scientists' experience of the process of writing research articles with focus on motivating factors and the feeling of success, in order to improve the process. Methods. This was a qualitative study using focus groups to explore young scientists' feelings and motivations regarding the process of writing scientific articles. Participants were young scientists including young medical doctors and medical students spending dedicated time on research. Content analysis was used to analyze the focus group interviews. Results. Sixteen informants participated in the study in three groups. Two major themes were identified: emotional support and setting and framework. Emotional support covered three subthemes: support from peers and supervisors, appearances, and motivation. The setting and framework theme covered four subthemes: deadlines, retreats, consciousness, and expectations. Conclusion. We found emotional support, frame-setting, and the avoidance of failures to be important factors for the feeling of success when young scientists are in the process of learning how to write scientific articles.
\end{abstract}

\section{Introduction}

Young medical scientists such as medical students conducting extracurricular research or Ph.D. students early in their career are considered highly specialized colleagues. Education and counseling can be complex and it is not clearly described how highly specialized coworkers are guided and motivated [1]. It has been increasingly common for Danish medical students to take a leave from their studies for a year and enroll in full time dedicated extracurricular research training. They often perform their own research projects and/or help with Ph.D. studies and most of them are expected to produce at least one scientific manuscript as first author [2]. Structuring of the time and which factors are needed in order to achieve the highest possible quality are often debated. Quality of the work as well as quality of the learning processes are areas that need attention. Scientists are expected to produce a high number of publications of high quality [3]. However, in many countries there is a lack of formal training and courses in this specialized task. Even though young scientists are highly specialized through their education, the process of writing articles is, for most of them, considered a new task and learning can be difficult. Learning is a process driven by the combination of body, mind, and experiences [4].

When supervisors train and teach young scientists to write articles, it is important to consider all aspects of learning. An important part is motivation, since scientific work can be demanding in a different way than routine clinical work. There can be a tendency among researchers to focus more on the final product such as the final accepted paper and less on the process. However, for the newcomer into this field, the process is considered a major challenge.

An institution such as a research group needs to be aware of the learning process and reflect on the assumptions regarding how young scientists can and should learn to perform research and write research articles. One way to establish a high level of motivation and commitment to this 
work is through the establishment of a community of practice in the research group [5]. Here the individual participants are likely to benefit from peers as well as more and less experienced colleagues, which can result in a greater learning outcome [5].

In regard to motivation four personality types can be identified [6]. The four types are described as the "prima donnas," the "achievement trippers," the "pragmatics," and the "workers." In general, persons in the field of research are likely to be either the prima donnas or the achievement trippers. These types are motivated by the "higher cause"; that is, the "kick" they get from achievements and credits for their work from supervisors and others. If supervisors are aware of these types and factors, they might be able to create a culture that will promote motivation and result in higher achievements and better learning output as well as high quality in the research product.

The focus of this study was to identify factors that supervisors should be aware of when planning and supervising the course of a research employment for young scientists. The aim of this study was to investigate young scientists' experience of the process of writing research articles with the focus on motivating and demotivating factors and the feeling of success, thereby providing insights to possible areas in need of attention.

\section{Materials and Methods}

This study was based on a qualitative design with the use of focus groups with a total of 16 informants [7] in order to explore young scientists' feelings and motivations regarding the process of writing scientific articles for scholarly journals. The study was performed and reported according to the Consolidated Criteria for Reporting Qualitative Research (COREQ) guidelines [8]. Focus groups were chosen as the methodology because it allows the participants to discuss and debate [9]. Furthermore, participants react and respond to the opinions and feelings expressed by other members of the group thereby opening for new insights into the process and phenomena [9].

Participants were sampled from a group of young scientists where the majority was enrolled in medical school. Potential participants were invited during a research course. The course was one of three annual writing retreats where the involved research groups meet and everyone dictates a full article manuscript draft [10-12]. Twice a year, the retreats are with one overnight stay and once a year the retreat covers a full week of writing and scientific tutoring. Participants were informed verbally and in writing. Participation was voluntary and informed consent forms were signed prior to the start of the study and withdrawal was accepted at any point without the need of stating a reason. Anonymity of all participants was ensured, and interviews were recorded, transcribed verbatim, and kept confidential. The group size ranged from five to six participants and new interviews were conducted until data saturation was reached. Data saturation was reached when no new themes emerged from the focus group interviews.
2.1. Data Collection. Focus group interviews were conducted in undisturbed surroundings, where participants sat comfortably and faced each other. A semistructured interview guide was developed a priori, based on three individual indepth interviews. The first author led the interviews and the second author acted as an observer as recommended for this type of data collection [13]. Participants were asked to share and discuss their feelings and thoughts about the process of writing research articles as well as the process of interacting in a research group. Each interview was approximately one hour or until the topic was covered to the satisfaction of participants and interviewers.

2.2. Data Analysis. Content analysis was used to analyze the transcribed interviews [14]. It provides the authors with the possibility and flexibility to develop and extend the themes on the background of meaning condensation and codes from the data. Analysis was conducted in parallel sessions and the first and second authors discussed findings and agreed on final themes. The interviews were first read in full in order to get an understanding of the interview as a whole. Then they were reread in order to find meaning units that were coded and divided into subthemes.

2.3. Ethical Considerations. Participants were informed both verbally and in writing prior to the study and provided informed consent. Data were anonymized and the study was approved by the Danish Data Protection Agency (HGH-2016061). According to Danish law ethical committee approval was not needed for this type of study.

\section{Results}

A total of 16 informants participated in the study in three groups. Participants had a median age of 26 years (range 23 to 35 ) and eight were women. Thirteen were medical students with median two years left of university studies and three were postgraduate medical Ph.D. students. Fulltime engagements in research varied from four months to two years with median one year. The participants had produced median 2 (range 1 to 6) final article drafts. The groups were sampled so participants had similar levels of experiences, but with a mix of sexes. Since the majority of participants came from three individual research groups, a mix between research groups was also ensured in order to avoid that one group solely discussed the experience from their own group. Data saturation was reached after two group interviews but a third one was added in order to fully ensure data saturation.

Two major themes were identified: emotional support and setting and framework. Emotional support covers three subthemes: support from peers and supervisors, appearances, and motivation. The setting and framework theme covered four subthemes: deadlines, retreats, consciousness, and expectations (Table 1).

3.1. Emotional Support. The support from peers and supervisors was needed for motivation and enhancing the feeling of success. As expressed by an informant: "Support from 
TABLE 1: Findings, major themes, and subthemes.

\begin{tabular}{lc}
\hline Major themes: & Subthemes \\
\hline \multirow{2}{*}{ Emotional support } & Support from peers and supervisors \\
& Appearances \\
& Motivation \\
\hline Setting and framework & Deadlines \\
& Retreats \\
& Consciousness \\
& Expectations \\
\hline
\end{tabular}

supervisors is important, they know how the process is, but it also helps to see peers work - that can be very motivating."

Support from supervisors and peers motivated and encouraged the participants in their own work. Seeing other participants of the research group succeed with their work such as the acceptance of research articles, approval from various authorities, or managing to get through a long to do list left the participants with comfort and a trust in succeeding. As expressed by a participant: "Regarding motivation - it does help to see others work, it can be very motivating, and you get inspired seeing others work."

Being part of a research group enhanced their motivation, especially when they found that peers also were stressed and experienced hard times. These feelings improved the sense of belonging, which was essential for the young scientist to be able to keep motivated and perform on a high level. The experience of seeing failure as a natural part of the scientific work encouraged them to keep working. They could see that peers were in the same situation and that they had managed to get through it. "When you are told that the situation you are in is normal, it helps because you are not used to the research world."

Appearances were often found to be two-faced. The participants described that the dynamic in the group was often driven by high performance and a high degree of competence. This dynamic seemed to be a set of values that all group members were committed to. The participants sometimes felt demotivated when they saw a high level of workflow, motivation, and energy set by their peers, when they felt unable to do the same. They described how they would hide it from the others in order not to show their weakness. When seeing the apparent high level of work and commitment from their peers and supervisors, they were sometimes left with the feeling of failure and they felt unable to perform on the same level. It had a detrimental effect on their motivation. However, it could be counteracted by discussion and realization that it was maybe just appearance and not reality that their peers as well supervisors presented. The appearances of high performance made them feel less competent. A way to get through was if some of their peers expressed their frustration and shared their experiences of feeling stressed or lost in the beginning. As one informant said: "I get frustrated, when I cannot deliver at the same level as more experienced peers. I know it's stupid, but I always feel I'm behind. I want to perform at a high level; it is so frustrating when I can't." And also: "When you are told that PhD students can dictate a PhD thesis, you feel like an idiot, when you can barely write one article."

A research group with a mixture of competence and experience was regarded as a motivating and supporting factor. It was motivating to be able to seek help and support from peers sitting in the same office. They also found it very motivating to experience that they themselves could help others and thereby realizing their own increase in skills and competences. The three research groups have prescheduled writing retreats three times a year. The participants expressed these retreats to be a motivating factor because everyone was working towards the same goal of writing scientific articles and everyone had the same deadline. As expressed by a young scientist: "Retreats can be stimulating for the process and the motivation. You have the time for it, you are on a deadline and you have a research group with different competences, where you can get support."

3.2. Major Theme: Setting and Framework. Writing a scientific article is considered a time-consuming process. Before the retreats, the participants are asked to make an outline of their manuscript and this process was surprisingly timeconsuming for the participants. They were surprised by their own need for guidance, before the retreat: "It is nice when your supervisors are clear on setting the process. Otherwise it's difficult to know what you are in charge of, what's your responsibility. It's often more time-consuming than you have expected."

In general, the scientific work, gathering of data, writing protocols, obtaining permissions from authorities, and writing research articles were all tasks that were more timeconsuming than initially thought by the participants. They expressed needs for guidance in time management and in realistic planning in order not to get frustrated when they could not reach their deadlines, often because they were too optimistic. "In the beginning you need tight guiding from you supervisor, you have no idea of the process and how long it takes. It's motivating when you in the beginning have talked the process through."

More conservative and realistic timelines set in collaboration with the supervisor could result in higher feeling of success. Deadlines were motivating and helped in reaching milestones in the research process: "It's important for me to have deadlines; otherwise it easily gets blurry for me." Deadlines were in general considered a good thing because it motivated participants to reach the goal and finalize their work. However, deadlines were often too few, and larger tasks would have to be completed for each deadline. Participants expressed a desire for frequent deadlines, with minor tasks for each deadline and thereby more often the feeling of reaching a goal. The deadlines should be used to set the framework for their work in collaboration with their supervisor, which should have the overview of the research process and the experience needed to make realistic plans. This would help in avoiding timeslots where no work was needed but also in avoiding the overoptimistic planning of time and thereby failure to reach the goals. "It is important to have supervisors 
TABLE 2: Point of focus for supervisors of young scientists.

\begin{tabular}{lc}
\hline Theme & Point of focus \\
\hline Emotional support & $\begin{array}{c}\text { Ensure an open discussion of difficulties and feeling of inadequacies among young researchers. } \\
\text { Ensure an environment where people are not showing false appearances of success and work without problems. }\end{array}$ \\
\hline Setting and framework & $\begin{array}{c}\text { Actively use frequent deadlines in order to ensure progress in the work and the feeling of accomplishments. } \\
\text { Retreats should be used as deadlines as well as providing time for the task of writing scientific articles. } \\
\text { Invest time in aligning expectations in relation to time management and how difficult research can be. }\end{array}$ \\
\hline
\end{tabular}

that help you set realistic goals and help you plan the process to avoid getting frustrated and demotivated."

The retreats (with a fixed deadline) were generally considered a good thing, because they provided allocated time to only focus on one task: the drafting of a scientific manuscript. However, there was a high need for guidance leading up to these major deadlines, because the participants were not experienced enough to plan it themselves. Thus, there was an unmet need for frequent deadlines that could result in a smother process. Furthermore, an earlier start of some of the project parts would help them to not feel frustrated and on the edge of giving up, because they felt like a failure and got too stressed in the last weeks or days leading up to the retreats.

Participation in the retreats and the drafting of manuscripts are clear expectations from supervisors in the research groups. However, there is a need to balance as well as have open discussions about these expectations. Sometimes the participants felt lost or not knowing whether they fulfilled the expectations in order to get the credit that they seek.

These findings can be used actively when supervising young scientists (Table 2).

\section{Discussion}

This qualitative study using focus group interviews with young scientists identified two major themes: emotional support and setting and framework. Each major theme covered several subthemes. The participants reported that they were in need of emotional support when writing articles and conducting research. They needed compliments as well as encouragement from both peers and supervisors in order to keep or heighten their motivation and increase the quality as well as the quantity of their research and article output. They also reported the need of a clear and well defined framework set forth by the supervisors in a dialogue with the young researcher. This was important in order to get an understanding of the timeline, have acceptable deadlines, and avoid bad consciousness. It was thought to be possible through balancing of expectations and understanding of the expectation set forth by both the supervisor and the young researcher.

The emotional support was important in order to heighten the motivation among the young scientists. The $\mathrm{X}$ and $\mathrm{Y}$ theory describes two archetypes of employees [15]. The X-types are described as being lazy and their reason for working is the need and ability to sustain themselves. The Y-types are motivated by the "higher cause" and its internal factors such as credit for their work and acknowledgements for achievements that motivate them in their work. They want to "push" themselves forward. Based on the findings in this study, young scientists most likely belong to the Ytype and therefore are in need of acknowledgement and credit for their work in order to keep motivation high. They can also be described as "prima donnas" or "achievement trippers" that are two kinds of personality that have the need for acknowledgements from others and need a feeling of success on a regular basis through their work to keep a high motivation. It is important that the supervisors are aware of these factors when giving feedback to the students. There is a paradox in the need and urge for feedback because the students are eager to get the feedback in order to learn new fields of research and the process of writing articles. However, they can be demotivated by feedback if it is not followed by some kind of credit [16]. If the young scientists are not led in a way which stimulates their motivation and stimulates them in their environment, there is a risk that they will change from X-type to Y-type, meaning that they will only fulfill the minimum of what is expected of them in order to conduct research and write articles [15]. Then they might not develop the skills required for a career in science and research. However, it is the intent of most supervisors to have young scientists that both get the job done and at the same time are reaching new learning levels and in the future be the ones to take research forward. If the motivation is not actively brought into the process of teaching people how to write articles they might end up being frustrated.

The emotional support also includes discussions and openness about times where the young scientist might be frustrated about the work because they get a feeling of wasting their time or not being able to fulfill the expectations. However, these feelings are normal in the process of learning to write scientific articles and conduct research and it should therefore be explicitly stated that this is a natural part of the process and that it is actually beneficial for the learning process, because these feelings can stimulate and enhance the learning output $[6,17]$.

The informants found it beneficial to be part of a research group. Being part of the research group meant that they were together with researchers that were less experienced and those who had the same level of experience as well as more experienced researchers and all shared the same goal of writing great articles. The community in which they conducted their daily work could both be stimulating and challenging, because they sometimes experienced a feeling that they were the dumbest of them all. However, it would 
also be supportive because they found support in the work of others and in seeing other people succeed. Being part of a community of practice does not necessarily mean a peaceful coexistence among people but it is a community functioning well with challenges as well as critical debates, frustrations, and emotional engagement [5]. There is an important task for experienced participants in the community of practice as well as supervisors in order to guide the new and young entering the community. The expectations and the daily participation in the community of practice could be fruitful in order for new and young researchers to become full members and enhance their learning output as much as possible as well as being able to provide useful insights and discussions within the community of practice [18].

Strength of a qualitative study especially in focus group studies can be found in the number of participants and interviews. Data saturation was reached with this large group of people interviewed. There are limitations to this study, one being that it was only young scientists that had already been trained in the same way of drafting and writing articles. Another is that the first and second authors who led the interviews were both part of these groups, although not directly working with any of the informants, and their presence during the interviews might have affected what was said and expressed. However, these limitations are unlikely to affect the overall results and findings.

In conclusion, this qualitative study using focus group interviews with young scientists found that emotional support and frame-setting were important factors for the feeling of success and the avoidance of failures when young scientists are in the process of learning how to write scientific articles. Young scientists as well as supervisors need to have open discussions in order to balance their expectations, discuss the process, and plan deadlines and expectations carefully. Through planning and discussion the motivation can be enhanced and the feeling of success can be strengthened thereby increasing the learning output as well as the output and the quality of the final product, that is, the research articles.

\section{Conflicts of Interest}

The authors declare that there are no conflicts of interest regarding the current manuscript.

\section{Authors' Contributions}

Laursen J, Andresen K, and Rosenberg J were involved in conception and design. Laursen $\mathrm{J}$ and Andresen $\mathrm{K}$ acquired the data. Laursen J, Andresen K, and Rosenberg J were responsible for analysis and interpretation of data. Laursen J, Andresen K, and Rosenberg J were responsible for drafting and revision. All authors provided their approval for the current version to be published.

\section{Acknowledgments}

The authors thank all the participants for their willingness to share their thoughts and ideas with them for this project.

\section{References}

[1] F. J. McArthur-Rouse, "From expert to novice: An exploration of the experiences of new academic staff to a department of adult nursing studies," Nurse Education Today, vol. 28, no. 4, pp. 401408, 2008.

[2] S. B. Andersen, L. Ostergaard, and P. L. Fosbol, "Extracurricular scientific production among medical students has increased in the past decade," Danish Medical Journal, vol. 62, no. 9, 2015.

[3] A. Y. Gasparyan, B. Nurmashev, A. A. Voronov, A. N. Gerasimov, A. M. Koroleva, and G. D. Kitas, "The pressure to publish more and the scope of predatory publishing activities," Journal of Korean Medical Science, vol. 31, no. 12, pp. 1874-1878, 2016.

[4] L. Morrice, "The learning migration nexus: towards a conceptual understanding," European Journal for Research on the Education and Learning of Adults, vol. 5, no. 2, pp. 149-159, 2014.

[5] S. Seibert, "The Meaning of a Healthcare Community of Practice," Nursing Forum, vol. 50, no. 2, pp. 69-74, 2015.

[6] H. H. Hein, "The motivation and management of highly specialized creative employees," Understanding Organizations in Complex, Emergent and Uncertain Environments, pp. 167-184, 2012.

[7] T. Freeman, "'Best practice' in focus group research: Making sense of different views," Journal of Advanced Nursing, vol. 56, no. 5, pp. 491-497, 2006.

[8] A. Tong, P. Sainsbury, and J. Craig, "Consolidated criteria for reporting qualitative research (COREQ): a 32-item checklist for interviews and focus groups," International Journal for Quality in Health Care, vol. 19, no. 6, pp. 349-357, 2007.

[9] B. Halkier, "Focus groups as social enactments: Integrating interaction and content in the analysis of focus group data," Qualitative Research, vol. 10, no. 1, pp. 71-89, 2010.

[10] J. Rosenberg, J. Burcharth, H. C. Pommergaard, and A. K. Danielsen, "Mind-to-paper is an effective method for scientific writing.," Danish Medical Journal, vol. 60, no. 3, p. A4593, 2013.

[11] L. Spanager, A. K. Danielsen, H.-C. Pommergaard, J. Burcharth, and J. Rosenberg, "A feeling of flow: Exploring junior scientists' experiences with dictation of scientific articles," BMC Medical Education, vol. 13, no. 1, article no. 106, 2013.

[12] H. C. Pommergaard, C. Huang, and J. Burcharth, "Voice recognition software can be used for scientific articles," Danish Medical Journal, vol. 62, no. no A5012, 2015.

[13] A. Fontana and J. H. Freuy, The Art of Science, Handbook of Qualitative Research, Sage Publications, 1994, 361-376.

[14] U. H. Graneheim and B. Lundman, "Qualitative content analysis in nursing research: concepts, procedures and measures to achieve trustworthiness," Nurse Education Today, vol. 24, no. 2, pp. 105-112, 2004.

[15] T. A. Erkiliç, "A discussion on the application of two factor, $\mathrm{x}$ and y theories in classroom management," American-Eurasian Journal of Scientific Research, vol. 3, pp. 111-116, 2008.

[16] E. A. Fisher, "Motivation and leadership in social work management: A review of theories and related studies," Administration in Social Work, vol. 33, no. 4, pp. 347-367, 2009.

[17] P. Jarvis, "Lifelong education and its relevance to nursing," Nurse Education Today, vol. 7, no. 2, pp. 49-55, 1987.

[18] A. Garrow and S. Tawse, "An exploration of the assessment experiences of new academics as they engage with a community of practice in higher education," Nurse Education Today, vol. 29, no. 6 , pp. 580-584, 2009. 


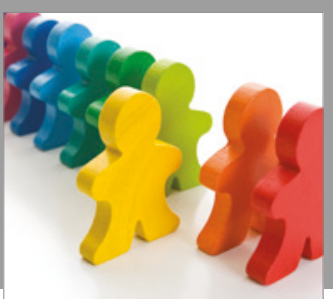

Autism

Research and Treatment
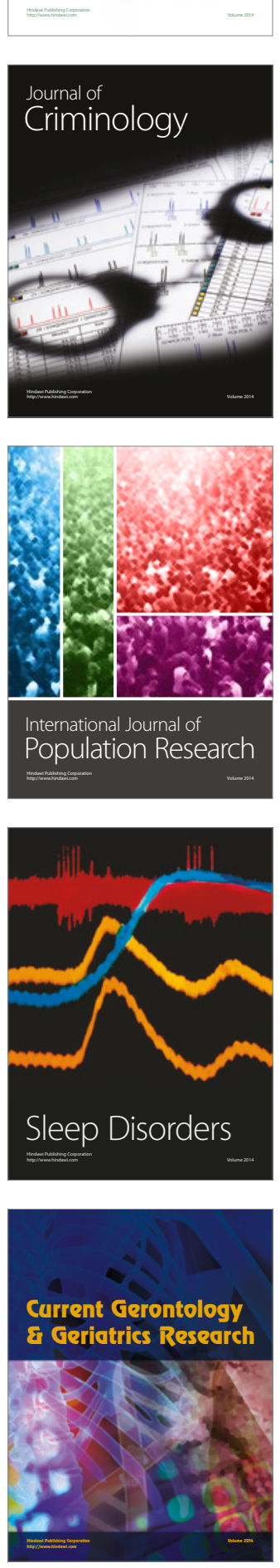

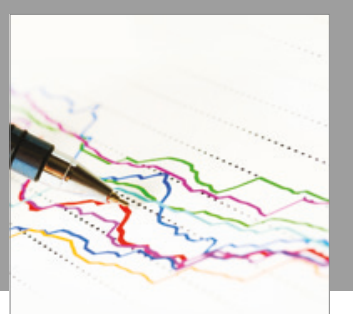

Economics

Research International

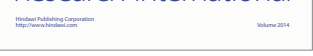

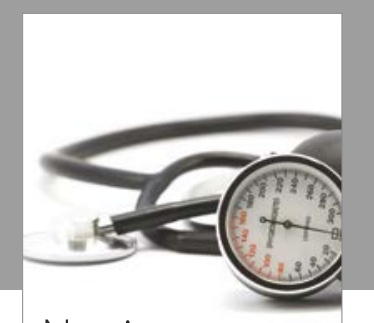

Nursing

Research and Practice

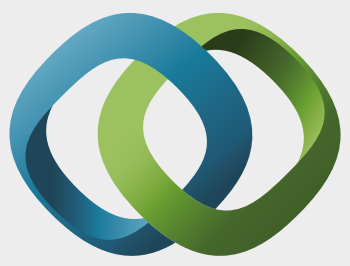

\section{Hindawi}

Submit your manuscripts at

https://www.hindawi.com
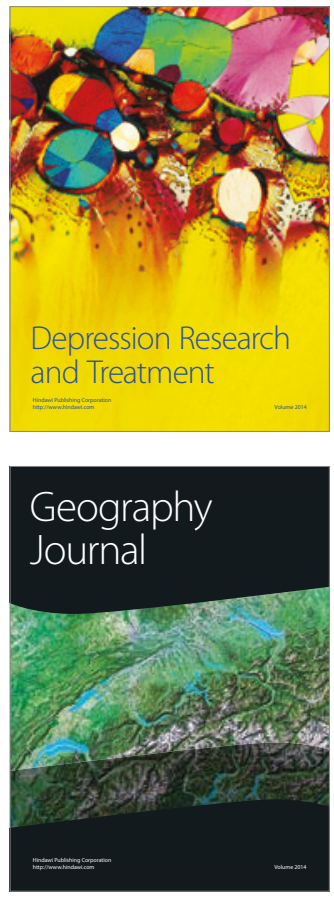
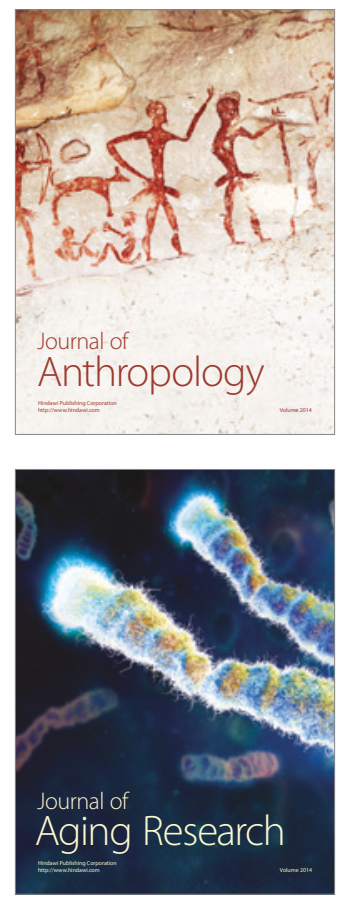
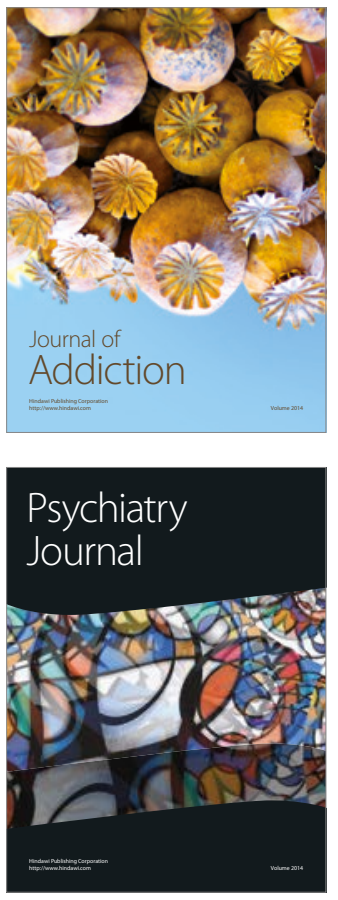

Child Development

Research

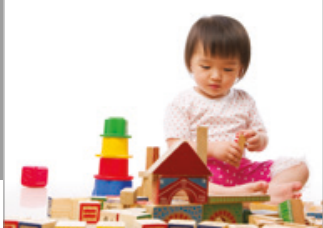

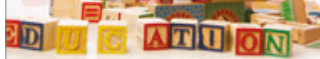
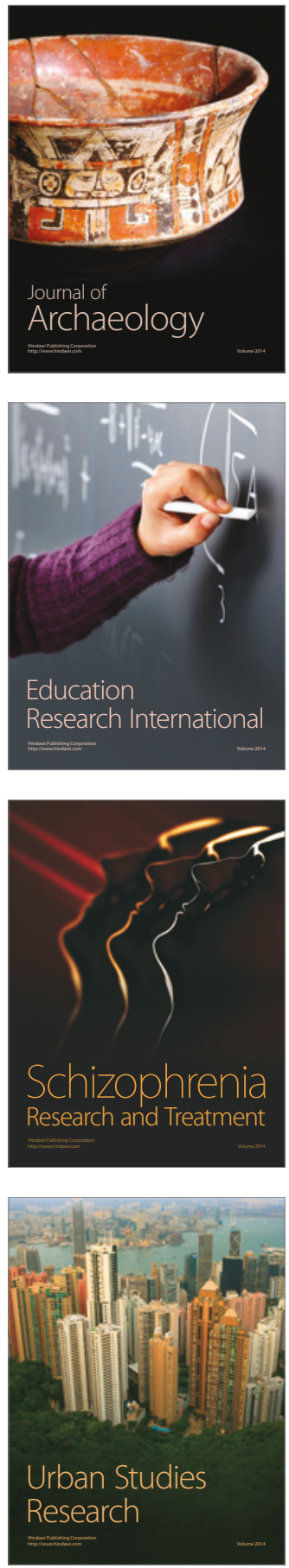